\title{
Centers for Medicare \& Medicaid Services Price Publication Requirement: If You Post It, Will They Come?
}

\author{
Jennifer B Cowart, MD ${ }^{1 \star}$, Suparna Dutta, MD, MPH 2,3
}

'Division of Hospital Internal Medicine, Department of Internal Medicine, Mayo Clinic, Jacksonville, Florida; ${ }^{2}$ Department of Medicine, Hartford Hospital, Hartford, Connecticut; ${ }^{3}$ University of Connecticut School of Medicine, Farmington, Connecticut.

atients in the United States continue to experience rising out-of-pocket medical costs, with little access to the price information they desire when making decisions regarding medical care. ${ }^{1}$ The Centers for Medicare \& Medicaid Services (CMS) has taken steps toward transparency by requiring hospitals to publish price information. ${ }^{2}$ In this issue of the Journal of Hospital Medicine, White and Liao ${ }^{3}$ break down the new rule, and we further discuss how this policy affects patients, hospitals, and hospitalists.

The new CMS rule requires hospitals to publish the prices of 300 "shoppable" services, including those negotiated with different payors. The rule standardizes how this information is displayed and accessed, with a daily penalty for facilities that fail to comply. Clinics and ambulatory surgical centers are currently excluded, as are facility and ancillary fees, such as those billed by pathology or anesthesiology. As White and Liao point out, a limitation for hospitalists is that this rule will only affect orders for the outpatient setting at discharge. In addition, this rule separates cost from quality. Although quality data are publicly available via CMS, price data are posted directly by hospitals, making a true value assessment difficult. To strengthen the rule, White and Liao recommend the following: increasing the financial penalty for noncompliance; aggregating data centrally to allow for comparisons; adding quality data to cost; expanding included sites and types of services; and adding common additional fees to the service price.

The larger question is whether patients will use these data in the manner intended. Previous studies have found a paradoxical relationship between patients' expressed desire to compare prices for medical services vs documented low levels of price-shopping behavior. Mehrotra et al ${ }^{1}$ found that lack of access to data as well as loyalty to providers were significant barriers to using price data effectively. The CMS rule increases access to the price information patients desire but cannot find. However, it is unclear whether available prices will be sufficient to change behaviors given that, aside from those with no insur-

*Corresponding Author: Jennifer B Cowart, MD;

Email: cowart.jennifer@mayo.edu; Telephone: 904-956-0081;

Twitter: @jbcowartmd.

Received: August 31, 2021; Accepted: September 3, 2021

○ 2021 Society of Hospital Medicine DOI 10.12788/jhm. 3708 ance and those with high-deductible plans, most patients are fairly removed from the actual cost of service.

This rule may have a larger, unexpected impact on hospitals and access to care. Sharing price data could increase pressure on facilities to merge with larger systems in order to obtain more favorable rates via increased negotiating power. Hospitals that serve poorer communities may not be attractive merger candidates for large systems and could be left out of the push toward consolidation. Charging higher prices for the same services could lead to hospital closures or cuts in resources, potentially exacerbating health inequities for underserved populations.

On the provider end, it is unlikely that price transparency will influence resource utilization. Mummadi et al ${ }^{4}$ found that displaying price information in the electronic health record did not significantly influence physician ordering behavior. For hospitalists today, the emphasis on "high-value care" is already an important consideration when utilizing healthcare resources, considering the Accreditation Council for Graduate Medical Education (ACGME) requirements for residency, restrictive insurance protocols, and guidelines such as the ACR Appropriateness Criteria and the American Board of Internal Medicine's Choosing Wisely ${ }^{\circledR}$ campaign. Outside of extremes, separate cost data likely will not make a difference in provider ordering practices.

Although the information from this rule may not cause dramatic practice change, it will allow us to help our patients by providing those interested in price-shopping with data. This policy represents a large step toward a more transparent healthcare system, though it may have limited impact on overall healthcare costs.

Disclosures: The authors reported no conflicts of interest.

\section{References}

1. Mehrotra A, Dean KM, Sinaiko AD, Sood N. Americans support price shopping for health care, but few actually seek out price information. Health Aff (Millwood). 2017;36(8):1392-1400. https://doi.org/10.1377/hlthaff.2016.1471

2. Price Transparency Requirements for Hospitals to Make Standard Charges Public. 45 CFR $\S 180.20$ (2019).

3. White AA, Liao JM. Policy in clinical practice: hospital price transparency. J Hosp Med. 2021;16(11):688-690. https://doi.org/10.12788/jhm.3698

4. Mummadi SR, Mishra R. Effectiveness of provider price display in computerized physician order entry (CPOE) on healthcare quality: a systematic review. J Am Med Inform Assoc. 2018;25(9):1228-1239. https://doi.org/10.1093/ jamia/ocy076 